06

\title{
Гибридный двухкомпонентный бризер
}

\author{
(ㄷ Г.Т. Адамашвили \\ Грузинский технический университет, \\ 0160 Тбилиси, Грузия \\ e-mail: guram_adamashvili@ymail.com \\ Поступила в редакцию 06.04.2021 г. \\ В окончательной редакции 27.04.2021 г. \\ Принята к публикации 26.05.2021 г.
}

\begin{abstract}
Рассмотрено нелинейное когерентное взаимодействие света с диспергирующей и керровской средой, содержащей оптические примесные атомы или полупроводниковые квантовые точки. Используя обобщенную версию метода пертурбативного разложения, нелинейное волновое уравнение сводится к связанным нелинейным уравнениям Шредингера. Показано, что производные второго порядка играют существенную роль в описании процесса образования связанного состояния двух бризеров, которые осциллируют на суммарной и разностной частотах и волновых числах. Резонансные, нерезонансные и гибридные механизмы формирования двухкомпонентного бризера реализуются в зависимости от параметров света и вещества. Приведены явные аналитические выражения для профиля и параметров нелинейного импульса. Обсуждены условия формирования резонансных, нерезонансных и гибридных нелинейных волн. В частном случае резонансный векторный бризер совпадает с векторным 0л-импульсом самоиндуцированной прозрачности.
\end{abstract}

Ключевые слова: гибридные нелинейные волны, самоиндуцированная прозрачность, двухкомпонентный бризер.

DOI: $10.21883 /$ OS.2021.08.51202.2128-21

\section{1. Введение}

Нелинейное взаимодействие света с веществом является областью интенсивных исследований, которая охватывает широкий класс оптических нелинейных явлений. При нелинейном когерентном взаимодействии света с веществом проявляются различные нелинейные процессы. Среди них особый интерес вызывают процессы, приводящие к образованию нелинейных уединенных волн устойчивого профиля. Существование этих волн является одним из самых ярких проявлений нелинейности в оптических средах. Выявление механизмов, вызывающих формирование оптических нелинейных уединенных волн, определение видов этих волн и изучение их свойств в различных нелинейных средах относятся к основным задачам нелинейной оптики.

В зависимости от характера нелинейности рассматриваются нерезонансный и резонансный механизмы образования нелинейных волн. В случае нерезонансной нелинейности, который связан с квадратичной и/или кубической нелинейной восприимчивостями, их конкуренция с дисперсионными или дифракционными эффектами может привести к формированию нерезонансных оптических нелинейных волн. Эти нелинейные волны классифицируют как временные или пространственные уединенные волны соответственно [1-3]. Резонансные оптические нелинейные волны могут формироваться в условиях самоиндуцированной прозрачности (СИП) [4-6].

Как резонансные, так и нерезонансные нелинейные уединенные волны делятся на два основных типа: однокомпонентные и двухкомпонентные нелинейные волны.
В основном к однокомпонентным нелинейным уединенным волнам относятся скалярные солитоны и скалярные бризеры, в то время как к двухкомпонентным волнам относятся векторные солитоны и векторные бризеры или их модификации.

Хотя явление СИП и резонансные нелинейные волны, а именно скалярные солитоны и скалярные бризеры, изучаются достаточно длительное время, в последние годы интерес к этим волнам значительно возрос. Проведено множество теоретических и экспериментальных исследований [7-13].

В теории СИП производные второго порядка по пространственной координате и времени напряженности электрического поля импульса в волновом уравнении Максвелла обычно не учитывались. В результате считалось, что основными волнами СИП являются скалярный однокомпонентный бризер (0л-импульс) и скалярный однокомпонентный солитон $(2 \pi$-импульс Мак-Колла и Хана) [4], которые можно рассмотреть при условии, когда в уравнениях СИП производными второго порядка можно пренебречь. Однако ситуация недавно изменилось в связи с развитием математического подхода, а именно обобщенной версии метода пертурбативного разложения (МПР) $[14,15]$. В отличие от стандартного МПР [16], адаптированного для исследования однокомпонентных нелинейных волн, обобщенный МПР позволяет перейти к следующему этапу развития теории СИП, а именно к исследованию свойств резонансных двухкомпонентных уединенных волн. Обобщенный МПР позволяет показать, насколько важную роль играют производные второго порядка в теории СИП. А именно 
учета первых производных в уравнениях СИП достаточно для описания однокомпонентных СИП-солитона и СИП-бризера, в то время как вторые производные в уравнениях СИП дают возможность описать взаимодействие между двумя однокомпонентными бризерами, которые приводят к образованию их связанного состояния. Такой двухкомпонентный бризер (ДБ) в теории СИП образует векторный $0 \pi$-импульс, который представляет собой связанное состояние двух бризеров идентичной поляризации, одна компонента которой осциллирует на суммарной, а вторая на разностной частотах и волновых числах (СРЧВ). В результате такой суперпозиции возникает нелинейный импульс нулевой площади со специфической фазовой модуляцией и профилем, который существенно отличается от профилей скалярного однокомпонентного СИП-солитона и скалярного однокомпонентного СИП-бризера. Следовательно, получается, что если не пренебрегать вторыми производными в уравнениях СИП, то основными импульсами СИП являются векторный $0 \pi$-импульс и скалярный $2 \pi$-импульс. А скалярный $0 \pi$-импульс СИП является лишь некоторым приближением векторного 0л-импульса, который имеет место только в том частном случае, если пренебречь производными второго порядка в уравнениях СИП, или в том случае, если использовать менее общий математический подход, чем обобщенный МПР, например, стандартный МПР.

Оптический резонансный векторный $0 \pi$-импульс СИП в изотропных и анизотропных материалах исследовался для однофотонных и двухфотонных резонансных переходов, для плоских и поверхностных волн, а также для волноводной моды в различных физических ситуациях [14,15,17-19]. Нерезонансным аналогом 0лимпульса является оптический нерезонансный ДБ, осциллирующий на СРЧВ, который был рассмотрен в диспергирующей среде керровского типа с использованием обобщенного МПР [20].

В зависимости от численных значений параметров света и среды может возникнуть такая физическая ситуация, когда одновременно действуют как резонансный, так и нерезонансный механизмы образования нелинейных волн. В этом случае реализуется гибридный (смешанный) механизм возбуждения нелинейной уединенной волны и может формироваться гибридный нелинейный импульс. Гибридные однокомпонентные скалярные солитоны и гибридные однокомпонентные скалярные бризеры исследовались различными математическими методами на протяжении многих лет [21-26]. Однако, используя обобщенный МПР, можно получить качественно новый результат, а именно гибридный ДБ, осциллирующий на СРЧВ, который до сих пор не рассматривался.

Целью настоящей статьи является: исследование процессов формирования оптического гибридного ДБ осциллирующего на СРЧВ, распространяющегося в диспергирующей и нелинейной среде керровского типа, содержащей малую концентрацию оптических примесных атомов или полупроводниковых квантовых точек (ПКТ); получение явных аналитических выражений для параметров гибридного ДБ, осциллирующего на СРЧВ в области несущей частоты и волнового числа; определение условий существования различных нелинейных волн.

Статья организована следующим образом: разд. 2 посвящен выводу уравнений СИП в диспергирующей среде керровского типа, содержащей оптически активные примесные атомы (ПКТ) для медленно меняющихся огибающих функций. В разд. 3 с помощью обобщенного МПР нелинейное волновое уравнение для огибающих функций преобразуется в связанные нелинейные уравнения Шредингера (НУШ) для вспомогательных функций. Получены явные аналитические выражения для профиля и параметров ДБ, осциллирующих на СРЧВ. В заключительном разд. 4 обсуждаются полученные результаты, роль производных второго порядка в процессе формирования связанного состояния двух волновых пакетов и рассматриваются критерии существования резонансных, нерезонансных и гибридных нелинейных волн.

\section{2. Вывод уравнений}

Рассмотрим процесс формирования оптического гибридного ДБ, осциллирующего на СРЧВ, в диспергирующей среде с керровской восприимчивостью третьего порядка, содержащей малую концентрацию $n_{0}$ оптически активных примесных атомов или ПКТ. Оптический линейно поляризованный вдоль оси $x$ импульс с длительностью $T \ll T_{1,2}$, частотой $\omega \gg 1 / T$ и волновым вектором $\mathbf{k}$ распространяется в положительном направлении оси $z$. Здесь $T_{1}$ и $T_{2}-$ времена продольной и поперечной релаксации оптических резонансных атомов или ПКТ соответственно. В общем случае независимо от конкретной природы дисперсии в анизотропных средах дисперсию можно охарактеризовать с помощью тензора диэлектрической проницаемости $\varepsilon_{i j}(z, t)$, который зависит от пространственной координаты $z$ и времени $t$, где $i, j=x, y, z$. Мы рассмотрим изотропную среду, в которой тензор диэлектрической проницаемости сводится к виду $\varepsilon_{i j}(z, t)=\varepsilon(z, t) \delta_{i j}$, где $\varepsilon(z, t)$ - скалярная функция, $\delta_{i j}$ - символ Кронекера.

Волновое уравнение для $x$-компоненты напряженности электрического поля $\mathbf{E}(E, 0,0)$ импульса имеет вид [27]

$$
C^{2} \frac{\partial^{2} E}{\partial z^{2}}-\frac{\partial^{2} D_{l}}{\partial t^{2}}=4 \pi \frac{\partial^{2} P}{\partial t^{2}},
$$

где $C-$ скорость света в вакууме,

$$
D_{l}=\int \varepsilon\left(z_{1}, t_{1}\right) E\left(z-z_{1}, t-t_{1}\right) d z_{1} d t_{1}
$$

- линейная часть $x$-компоненты вектора электрической индукции. Нелинейная поляризация среды $P=P_{n}+P_{r}$ 
содержит нерезонансную и резонансную части.

$$
\begin{aligned}
& P_{n}=\int \rho_{x x x x}\left(z_{1}, z_{2}, z_{3}, t_{1}, t_{2}, t_{3}\right) E\left(z-z_{1}, t-t_{1}\right) \\
& \times E\left(z-z_{1}-z_{2}, t-t_{1}-t_{2}\right) \\
& \times E\left(z-z_{1}-z_{2}-z_{3}, t-t_{1}-t_{2}-t_{3}\right) d z_{1} d z_{2} d z_{3} d t_{1} d t_{2} d t_{3}
\end{aligned}
$$

является $x$-компонентой нерезонансной нелинейной поляризацией среды керровского типа, $\rho_{x x x x}-$ компонента тензора восприимчивости. $P_{r}=n_{0} \mu s_{1}$ является $x$ компонентой вектора поляризации системы двухуровневых атомов или ПКТ, где $\mu$ - электродипольный момент для соответствующих квантовых переходов оптических атомов или ПКТ, $s_{i}(t)=\left\langle\hat{\sigma}_{i}(t)\right\rangle-$ средние значения операторов Паули $\hat{\sigma}_{i}(i=1,2,3)$.

Зависимость величины $P_{r}$ от напряженности электрического поля импульса $E$ определяется из уравнений Блоха $[6,28]$ :

$$
\begin{gathered}
\frac{\partial s_{1}}{\partial t}=-\omega_{0} s_{2}, \\
\frac{\partial s_{2}}{\partial t}=\omega_{0} s_{1}+\kappa_{0} E s_{3}, \\
\frac{\partial s_{3}}{\partial t}=-\kappa_{0} E s_{2},
\end{gathered}
$$

где $\kappa_{0}=\frac{2 \mu}{\hbar}, \hbar-$ постоянная Планка, $\omega_{0}-$ резонансная частота возбуждения примесных двухуровневых атомов или ПКТ.

Система уравнений (1)-(4) представляет собой систему уравнений СИП в диспергирующей керровской среде. Учитывая, что длительность импульса $T \gg \omega^{-1}$, эти уравнения можно упростить, используя метод медленно меняющейся огибающей. Для этой цели представим $x$ компоненты напряженности электрического поля импульса $E$ и поляризации $P_{r}$ в следующей форме [5]:

$$
E=\sum_{l= \pm 1} \hat{E}_{l} Z_{l}, \quad P_{r}=n_{0} \mu \sum_{l} d_{-l} Z_{l}
$$

где $\hat{E}_{l}$ и $d_{l}$ - медленно меняющиеся комплексные амплитуды электрического поля волны и поляризации оптически активных примесных двухуровневых атомов или ПКТ, $Z_{l}=e^{i l(k z-\omega t)}$. Учитывая, что функция $E$ вещественна, получаем условие $\hat{E}_{l}=\hat{E}_{-l}^{*}$. Комплексные огибающие $\hat{E}_{l}$ и $d_{l}$ меняются достаточно медленно по пространсвенной координате и времени по сравнению с быстро осциллирующей частью $Z_{l}$, т. е. имеют место неравенства

$$
\begin{gathered}
\left|\frac{\partial \hat{E}_{l}}{\partial t}\right| \ll \omega\left|\hat{E}_{l}\right|, \quad\left|\frac{\partial \hat{E}_{l}}{\partial z}\right| \ll k\left|\hat{E}_{l}\right|, \\
\left|\frac{\partial d_{l}}{\partial t}\right| \ll \omega\left|d_{l}\right|, \quad\left|\frac{\partial d_{l}}{\partial z}\right| \ll k\left|d_{l}\right| .
\end{gathered}
$$

Подставляя уравнение (5) в (2) и учитывая (6) мы получим

$$
\begin{aligned}
D_{l} & =\sum_{l} Z_{l}\left[\kappa-i a \frac{\partial}{\partial z}+i b \frac{\partial}{\partial t}\right. \\
& \left.-c \frac{\partial^{2}}{\partial z^{2}}-d \frac{\partial^{2}}{\partial t^{2}}+\tilde{t} \frac{\partial^{2}}{\partial z \partial t}\right] \hat{E}_{l},
\end{aligned}
$$

где

$$
\begin{gathered}
\kappa=\int \varepsilon\left(z_{1}, t_{1}\right) e^{-i l\left(k z_{1}-\omega t_{1}\right)} d z_{1} d t_{1}, \\
a=-i \int \varepsilon\left(z_{1}, t_{1}\right) z_{1} e^{-i l\left(k z_{1}-\omega t_{1}\right)} d z_{1} d t_{1}, \\
b=i \int \varepsilon\left(z_{1}, t_{1}\right) t_{1} e^{-i l\left(k z_{1}-\omega t_{1}\right)} d z_{1} d t_{1}, \\
c=-\int \varepsilon\left(z_{1}, t_{1}\right) \frac{z_{1}{ }^{2}}{2} e^{-i l\left(k z_{1}-\omega t_{1}\right)} d z_{1} d t_{1}, \\
d=-\int \varepsilon\left(z_{1}, t_{1}\right) \frac{t_{1}{ }^{2}}{2} e^{-i l\left(k z_{1}-\omega t_{1}\right)} d z_{1} d t_{1}, \\
\tilde{t}=\int \varepsilon\left(z_{1}, t_{1}\right) t_{1} z_{1} e^{-i l\left(k z_{1}-\omega t_{1}\right)} d z_{1} d t_{1} .
\end{gathered}
$$

Подставляя уравнения (3), (5) и (7) в волновое уравнение (1), получаем закон дисперсии импульса в среде

$$
C^{2} k^{2}=\omega^{2} \kappa
$$

и нелинейное волновое уравнение для комплексной огибающей функции $\hat{E}_{l}$ в форме:

$$
\begin{aligned}
& \sum_{l= \pm 1} Z_{l}\left\{\left[i g_{1} \frac{\partial \hat{E}_{l}}{\partial z}+i g_{3} \frac{\partial \hat{E}_{l}}{\partial t}+g_{2} \frac{\partial^{2} \hat{E}_{l}}{\partial z^{2}}-g_{5} \frac{\partial^{2} \hat{E}_{l}}{\partial t^{2}}\right.\right. \\
& \left.\left.-g_{4} \frac{\partial^{2} \hat{E}_{l}}{\partial z \partial t}\right]-4 \pi \omega^{2} \sum_{l^{\prime}} \sum_{l^{\prime \prime}} \tilde{\rho}_{l, l^{\prime}, l^{\prime \prime}} \hat{E}_{l^{\prime}} \hat{E}_{l^{\prime \prime}}\right\}+4 \pi \frac{\partial^{2} P_{r}}{\partial t^{2}}=0,
\end{aligned}
$$

где

$$
\begin{gathered}
g_{1}=\omega^{2} a-2 l k C^{2}, \quad g_{2}=\omega^{2} c-C^{2}, \\
g_{3}=-\left(\omega^{2} b+2 l \omega \kappa\right), \quad g_{4}=2 l \omega a+\omega^{2} \tilde{t}, \\
g_{5}=-\left(\omega^{2} d+2 l \omega b+\kappa\right) . \\
\tilde{\rho}_{l, l^{\prime}, l^{\prime \prime}}=\int \rho_{x x x x}\left(z_{1}, z_{2}, z_{3}, t_{1}, t_{2}, t_{3}\right) e^{-i l\left(k z_{1}-\omega t_{1}\right)} \\
\times e^{-i\left(l^{\prime}+l^{\prime \prime}\right)\left[k z_{2}-\omega t_{2}\right]} e^{-i l^{\prime \prime}\left[k z_{3}-\omega t_{3}\right]} d z_{1} d z_{2} d z_{3} d t_{1} d t_{2} d t_{3} .
\end{gathered}
$$

Уравнение (9) описывает различные нелинейные процессы, возникающие при нелинейном когерентном взаимодействии света с диспергирующей керровской средой, содержащей оптические примесные атомы (ПКТ). В частности, процессы образования однокомпонентных и двухкомпонентных уединенных нелинейных волн при условии, когда резонансный и нерезонансный механизмы формирования нелинейного импульса являются одновременно эффективными. 


\section{3. Двухкомпонентный нелинейный импульс}

Для анализа системы уравнений (4) и (9) можем использовать обобщенный МПР, предложенный в работах $[14,15,17-20]$. С помощью этого метода становится возможным трансформировать систему этих уравнений к СНУШ, который имеет решение в виде ДБ. В рамках этого метода комплексную функцию $\hat{E}_{l}(z, t)$ можно представить в следующем виде:

$$
\hat{E}_{l}(z, t)=\sum_{\alpha=1} \varepsilon^{\alpha} \hat{E}_{l}^{(\alpha)}=\sum_{\alpha=1}^{\infty} \sum_{n=-\infty}^{+\infty} \varepsilon^{\alpha} Y_{l, n} f_{l, n}^{(\alpha)}(\xi, \tau),
$$

где

$$
\begin{aligned}
Y_{l, n}=e^{i n\left(Q_{l, n} z-\Omega_{l, n} t\right)}, & \xi_{l, n}=\varepsilon Q_{l, n}\left(z-v_{g ; l, n} t\right), \\
\tau=\varepsilon^{2} t, & v_{g ; l, n}=\frac{\partial \Omega_{l, n}}{\partial Q_{l, n}},
\end{aligned}
$$

$\varepsilon$ - малый параметр. В разложении (10) выделяется из функции $\hat{E}_{l}$ еще более медленно меняющиеся функции $f_{l, n}^{(\alpha)}$. Следовательно, для величин $\Omega_{l, n}, Q_{l, n}$ и $f_{l, n}^{(\alpha)}$ при любых значениях индексов $l$ и $n$ предполагается выполнение следующих неравенств:

$$
\begin{aligned}
& \omega \gg \Omega_{l, n}, \quad k \gg Q_{l, n}, \\
& \left|\frac{\partial f_{l, n}^{(\alpha)}}{\partial t}\right| \ll \Omega_{l, n}\left|f_{l, n}^{(\alpha)}\right|, \quad\left|\frac{\partial f_{l, n}^{(\alpha)}}{\partial z}\right| \ll Q_{l, n}\left|f_{l, n}^{(\alpha)}\right| .
\end{aligned}
$$

В отличие от стандартного МПР [16] в разложении (10) мы рассматриваем ситуацию, когда величины $f_{l, n}^{(\alpha)}, Q_{l, n}$ и $\Omega_{l, n}$ зависят от индексов $l$ и $n$, тем самым увеличивая количество параметров, характеризующих волновой процесс.

Подставляя уравнения (5) и (10) в систему уравнений Блоха (4), получаем поляризацию двухуровневых атомов в виде

$$
\begin{aligned}
P_{r} & =i \frac{n_{0} \mu^{2}}{\hbar}\langle g\rangle \sum_{l= \pm 1} l Z_{l}\left[\varepsilon^{1} \Theta_{l}^{(1)}\right. \\
& +\varepsilon^{2} \Theta_{l}^{(2)}+\varepsilon^{3} \Theta_{l}^{(3)}-\varepsilon^{3} \frac{\kappa_{0}^{2}}{2} \\
& \left.\times \int \frac{\partial \Theta_{l}^{(1)}}{\partial t} \Theta_{-l}^{(1)} \Theta_{l}^{(1)} d t^{\prime}\right]+\mathscr{O}\left(\epsilon^{4}\right),
\end{aligned}
$$

где

$$
\begin{aligned}
\Theta_{l}^{(\alpha)}(z, t) & =\int_{-\infty}^{t} \hat{E}_{l}^{(\alpha)}\left(z, t^{\prime}\right) d t^{\prime}, \\
\langle g\rangle & =\int \frac{g(\Delta) d \Delta}{1+T^{2} \Delta^{2}},
\end{aligned}
$$

$g(\Delta)$ - нормированная функция неоднородного уширения спектральной линии для ансамбля двухуровневых оптических атомов или ПКТ, $\Delta=\omega_{0}-\omega, \alpha=1,2,3$.
Подставляя уравнения (10) в (9) и учитывая явный вид для поляризации (11), получим

$$
\begin{aligned}
& \sum_{l= \pm 1} \sum_{\alpha=1}^{\infty} \sum_{n=-\infty}^{+\infty} \varepsilon^{\alpha} Y_{l, n} Z_{l}\left\{W_{l, n}+i \varepsilon J_{l, n} \frac{\partial}{\partial \xi}+i \varepsilon^{2} h_{l, n} \frac{\partial}{\partial \tau}\right. \\
& \left.+\varepsilon^{2} H_{l, n} \frac{\partial^{2}}{\partial \xi^{2}}\right\} f_{l, n}^{(\alpha)}=\varepsilon^{3} \sum_{l= \pm 1} Z_{l} R_{l}\left[\left(\left|f_{l, l}^{(1)}\right|^{2}+2\left|f_{l,-l}^{(1)}\right|^{2}\right)\right. \\
& \left.\times f_{l, l}^{(1)} Y_{l, l}+\left(\left|f_{l,-l}^{(1)}\right|^{2}+2\left|f_{l, l}^{(1)}\right|^{2}\right) f_{l,-l}^{(1)} Y_{l,-l}\right] \\
& \left.-\varepsilon^{3} i \varkappa_{2} \sum_{l= \pm 1} l Z_{l} \int \frac{\partial \Theta_{l}^{(1)}}{\partial t} \Theta_{-l}^{(1)} \Theta_{l}^{(1)} d t^{\prime}+\ldots\right\}
\end{aligned}
$$

где

$$
\begin{gathered}
W_{l, n}=g_{3} n \Omega_{l, n}-g_{1} n Q_{l, n}-g_{2} Q_{l, n}^{2}+g_{5} \Omega_{l, n}^{2} \\
-g_{4} Q_{l, n} \Omega_{l, n}+\varkappa_{1} \frac{l n}{\Omega_{l, n}}, \\
J_{l, n}=g_{3} v_{g_{l, n}}-g_{1}-2 g_{2} n Q_{l, n}+2 n g_{5} \Omega_{l, n} v_{g_{l, n}} \\
-g_{4} n\left(Q_{l, n} v_{g_{l, n}}+\Omega_{l, n}\right), \\
h_{l, n}=g_{3}+2 g_{5} n \Omega_{l, n}-g_{4} n Q_{l, n}, \\
H_{l, n}=Q_{l, n}^{2}\left(g_{2}-g_{5} v_{g_{l, n}}^{2}+g_{4} v_{g_{l, n}}\right), \\
\varkappa_{1}=\frac{4 \pi \omega^{2} n_{0} \mu^{2}}{\hbar}<g>, \\
\varkappa_{2}=\frac{8 \pi n_{0} \mu^{4}}{\hbar^{3}}<g>, \\
R_{l}=4 \pi \omega^{2}\left(\tilde{\rho}_{l, l, l}+\tilde{\rho}_{l, l,-l}+\tilde{\rho}_{l,-l, l}\right) .
\end{gathered}
$$

Для определения функций $f_{l, n}^{(\alpha)}$ из уравнения (12) приравняем нулю различные члены при одинаковых степеньях малого параметра $\varepsilon$. В результате получаем цепочку уравнений. В первом порядке по $\varepsilon$ получаем, что из всех функциий $f_{l, n}^{(1)}$ отличны от нуля только функции: $f_{ \pm 1, \pm 1}^{(1)}$ или $f_{ \pm 1, \mp 1}^{(1)}$, а соотношение между параметрами $\Omega_{l, n}$ и $Q_{l, n}$ имеет следующий вид $(l= \pm 1, n= \pm 1)$ :

$$
\begin{aligned}
E(z, t)= & g_{3} n \Omega_{l, n}-g_{1} n Q_{l, n}+\varkappa_{1} \frac{l n}{\Omega_{l, n}} \\
& =g_{2} Q_{l, n}^{2}-g_{5} \Omega_{l, n}^{2}+g_{4} Q_{l, n} \Omega_{l, n} .
\end{aligned}
$$

Подставляя уравнения (14) в (12), во втором порядке по $\varepsilon$ мы можем доказать справедливость условий $J_{ \pm 1, \pm 1}=J_{ \pm 1, \mp 1}=0$.

Из уравнения (14) следует

$$
\begin{gathered}
\Omega_{+1,-1}=\Omega_{-1,+1}=\Omega_{-}, \quad \Omega_{+1,+1}=\Omega_{-1,-1}=\Omega_{+}, \\
Q_{+1,-1}=Q_{-1,+1}=Q_{-}, \quad Q_{+1,+1}=Q_{-1,-1}=Q_{+} .
\end{gathered}
$$


Уравнение (12), в третьем порядке по $\varepsilon$, в обозначениях $u_{ \pm 1}=\varepsilon f_{+1, \pm 1}$, трансформируются к связанным НУШ в форме

$$
\begin{aligned}
& i\left(\frac{\partial u_{ \pm 1}}{\partial t}+v_{ \pm} \frac{\partial u_{ \pm 1}}{\partial z}\right)+p_{ \pm} \frac{\partial^{2} u_{ \pm 1}}{\partial z^{2}} \\
& +q_{ \pm}\left|u_{ \pm 1}\right|^{2} u_{ \pm 1}+r_{ \pm}\left|u_{\mp 1}\right|^{2} u_{ \pm 1}=0
\end{aligned}
$$

где

$$
\begin{gathered}
v_{ \pm}=v_{g ;_{+1, \pm 1}}, \quad p_{ \pm}=\frac{H_{+1, \pm 1}}{h_{+1, \pm 1} Q_{ \pm}^{2}} \\
q_{ \pm}=\frac{R_{+1} \pm \frac{\varkappa_{2}}{\Omega_{ \pm}^{3}}}{h_{+1, \pm 1}}, \quad r_{ \pm}=\frac{2 R_{+1} \pm \frac{\varkappa_{2}}{\Omega_{ \pm}^{2} \Omega_{\mp}}\left(\frac{\Omega_{ \pm}}{\Omega_{\mp}}-1\right)}{h_{+1, \pm 1}}
\end{gathered}
$$

$u_{+1}$ и $u_{-1}-$ медленно меняющиеся комплексные функции огибающих волновых пакетов.

Из выражений (17) очевидно, что, так же как и для однокомпонентных гибридных волн [21-26], коэффициент $r_{ \pm}$для двухкомпонентых гибридных волн содержит аддитивный вклад от резонансной и нерезонансной нелинейностями.

Для решения уравнений (16) представим эти функции в следующей форме:

$$
u_{ \pm 1}(z, t)=\frac{f_{ \pm 1}}{b T} \operatorname{Sech}\left(\frac{t-\frac{z}{V_{0}}}{T}\right) e^{i\left(k_{ \pm 1} z-\omega_{ \pm 1} t\right)},
$$

где величины $f_{ \pm 1}, k_{ \pm 1}$ и $\omega_{ \pm 1}$ являются вещественными постоянными, $V_{0}$ - скорость нелинейной волны. При этом выполняются неравенства

$$
k_{ \pm 1} \ll Q_{ \pm}, \quad \omega_{ \pm 1} \ll \Omega_{ \pm} .
$$

Подставляя компоненты $u_{+1}$ и $u_{-1}$ из уравнения (18) в уравнения (16), (5) и (10) для $x$-компоненты электрического поля импульса $E(z, t)$, мы получим решение ДБ, осциллирующего на СРЧВ, в форме

$$
\begin{aligned}
E(z, t) & =\frac{2}{b T} \operatorname{Sech}\left(\frac{t-\frac{z}{V_{0}}}{T}\right)\left[f _ { + 1 } \operatorname { c o s } \left[\left(k+Q_{+}+k_{+1}\right) z\right.\right. \\
& \left.-\left(\omega+\Omega_{+}+\omega_{+1}\right) t\right]+f_{-1} \cos \left[\left(k-Q_{-}+k_{-1}\right) z\right. \\
& \left.-\left(\omega-\Omega_{-}+\omega_{-1}\right) t\right],
\end{aligned}
$$

где

$$
\begin{gathered}
T^{-2}=V_{0}^{2} \frac{v_{+} k_{+1}+k_{+1}^{2} p_{+}-\omega_{+1}}{p_{+}}, \\
b^{2}=V_{0}^{2} \frac{f_{+1}^{2} q_{+}+f_{-1}^{2} r_{+}}{2 p_{+}}, \quad k_{ \pm 1}=\frac{V_{0}-v_{ \pm}}{2 p_{ \pm}} .
\end{gathered}
$$

Соотношение между величинами $f_{ \pm 1}$ и $\omega_{ \pm 1}$ определяется из выражений

$$
\begin{gathered}
f_{+1}^{2}=\frac{p_{+} q_{-}-p_{-} r_{+}}{p_{-} q_{+}-p_{+} r_{-}} f_{-1}^{2} \\
\omega_{+1}=\frac{p_{+}}{p_{-}} \omega_{-1}+\frac{V_{0}^{2}\left(p_{-}^{2}-p_{+}^{2}\right)+v_{-}^{2} p_{+}^{2}-v_{+}^{2} p_{-}^{2}}{4 p_{+} p_{-}^{2}} .
\end{gathered}
$$

\section{4. Обсуждение результатов}

Мы рассматриваем когерентное нелинейное взаимодействие света с диспергирующей средой керровского типа, содержащей оптические примесные атомы или ПКТ. Показано, что наряду с исследованными ранее гибридными однокомпонентными нелинейными волнами (солитонами и бризерами) двухкомпонентная нелинейная волна (векторный бризер) может также формироваться при одновременном действии как резонансного, так и нерезонансного механизмов формирования нелинейных волн.

Выражение (20) является ДБ осциллирующим на СРЧВ, который является решением нелинейного волнового уравнения (1). Это выражение можно рассматривать как суперпозицию двух бризеров малой амплитуды. Первый член уравнения (20) - это бризер малой амплитуды $\left(f_{+1}\right)$, осциллирующий на суммарной частоте $\omega+\Omega_{+}$и волнового числа $k+Q_{+}$, а второе слагаемое - бризер малой амплитуды $\left(f_{-1}\right)$, осциллирующий на разностной частоте $\omega n-\Omega_{-}$и волнового числа $k-Q_{-}$(с учетом условия (19)). Нелинейная связь между этими двумя волновыми пакетами определяется перекрестными членами $r_{+}\left|u_{-1}\right|^{2} u_{+1}$ и $r_{-}\left|u_{+1}\right|^{2} u_{-1}$ уравнения (16). Оба бризера поляризованы вдоль оси $x$. Параметры бризеров тесно связаны между собой и определяются уравнениями (13), (17), (21) и (22). Эти бризеры образуют единый импульс (20), который распространяется в среде со скоростью $V_{0}$. Дисперсионное соотношение и связь между осциллирующими параметрами $\Omega_{ \pm}$и $Q_{ \pm}$определяются уравнениями (8) и (14). Подобно скалярному однокомпонентному солитону и однокомпонентному бризеру гибридный ДБ, осциллирующий на СРЧВ (20), не теряет энергию при распространении через среду. Ввиду того, что в разных физических системах величина $\varkappa_{2}$ в уравнении (13) изменяется в очень широком диапазоне, мы можем рассматривать три различные физические ситуации.

1. В среде, где выполняется условие $R_{+1} \ll \frac{\varkappa_{2}}{2 \Omega_{ \pm}^{2} \Omega_{\mp}}\left(\frac{\Omega_{ \pm}}{\Omega_{\mp}}-1\right), \quad$ формируется резонансный ДБ, осциллирующий на СРЧВ в условиях СИП. В этом случае ДБ совпадает с векторным 0л-импульсом СИП [14,15,17-19].

2. Нерезонансный ДБ, осциллирующий на СРЧВ, может быть сформирован, когда параметры света и среды удовлетворяют условию $R_{+1} \gg \frac{\chi_{2}}{2 \Omega_{ \pm}^{2} \Omega_{\mp}}\left(\frac{\Omega_{ \pm}}{\Omega_{\mp}}-1\right)$ [20].

3. Гибридный ДБ, осциллирующий на СРЧВ, может быть сформирован в ситуации, когда $R_{+1}$ и $\frac{\varkappa_{2}}{2 \Omega_{ \pm}^{2} \Omega_{\mp}}\left(\frac{\Omega_{ \pm}}{\Omega_{\mp}}-1\right)$ являются величинами одного порядка.

Из сравнения (6) и (9) очевидно, что производные второго порядка $g_{2} \frac{\partial^{2} \hat{E}_{l}}{\partial z^{2}}-g_{5} \frac{\partial^{2} \hat{E}_{l}}{\partial t^{2}}-g_{4} \frac{\partial^{2} \hat{E}_{l}}{\partial z \partial t}$ меньше, чем производные первого порядка $i\left(g_{1} \frac{\partial \hat{E}_{l}}{\partial z}+g_{3} \frac{\partial \hat{E}_{l}}{\partial t}\right)$. Тем не менее производные второго порядка играют существенную роль в процессе образования нелинейных уединенных волн. Действительно, производные 
второго порядка уравнения (9) связаны с членами $g_{2} Q_{l, n}^{2}-g_{5} \Omega_{l, n}^{2}+g_{4} Q_{l, n} \Omega_{l, n}$ уравнения (14) и с характеризующими осцилляции параметрами $\Omega_{ \pm}$и $Q_{ \pm}$в уравнении (15).

В частном случае, если пренебречь производными второго порядка в уравнении (9), как это часто делалось до разработки обобщенного МПР, то мы можем получить скалярное однокомпонентное СИП-солитонное и скалярное однокомпонентное СИП-бризерное решения уравнения (9). Действительно, в этом случае, если пренебречь в уравнении (14) членами с коэффициентами $g_{2}$, $g_{4}$ и $g_{5}$, мы видим, что это уравнение больше не зависят от индексов $l$ и $n$, учитывая, что коэффициенты $g_{1}$ и $g_{3}$ пропорциональны $l$. Следовательно, выполняются условия $\Omega_{+}=\Omega_{-}, Q_{+}=Q_{-}$и $r_{ \pm}=0$ и резонансный ДБ, осциллирующего на СРЧВ (случай 1), распадается на два скалярных однокомпонентных СИП-бризера, распространяющихся независимо друг от друга.

Отметим, что в теории СИП можно пренебречь производными второго порядка, поскольку они не участвуют в процессе формирования однокомпонентных СИПимпульсов, для описания которых достаточно учета производных первого порядка, а участвуют только в процессе формирования связанного состояния двух бризеров.

Для нерезонансного импульса (случай 2) ситуация иная. Производные второго порядка в уравнении (9) не только участвуют в образовании связанного состояния двух бризеров, но также участвуют в процессе формирования как однокомпонентной, так и двухкомпонентной нерезонансной нелинейной уединенной волн. Это объясняется тем, что для образования нерезонансной нелинейной уединенной волны необходимо, чтобы керровская нелинейность компенсировалась дисперсией (дифракционные эффекты здесь не рассматриваются). Эффекты дисперсии связаны с членом $H_{l, n}$ в уравнении (13), и если пренебречь членами второго порядка в уравнении (9) с коэффициентами $g_{2}, g_{4}, g_{5}$, мы видим, что величина $H_{l, n}$ становится равной нулю и нерезонансная уединенная волна не образуется. Итак, когда мы исследуем нерезонансные нелинейные уединенные волны в отличие от теории СИП (случай 1), мы всегда должны учитывать производные второго порядка в волновом уравнении (9).

Для гибридного ДБ, осциллирующего на СРЧВ (случай 3), ситуация аналогична нерезонансному случаю, поскольку для гибридных волн как резонансные, так и нерезонансные механизмы участвуют в формировании нелинейных уединенных волн одновременно, следовательно, мы должны сохранить производные второго порядка в волновом уравнении (9).

Следует также отметить еще одно важное обстоятельство, касающееся производных второго порядка в волновом уравнении (9). Хотя мы можем сохранить производные второго порядка в уравнении (9), тем не менее мы не всегда можем получить ДБ, осциллирующего на СРЧВ (см., например, [25]). Это происходит потому, что для исследования двухкомпонентных уединенных волн необходимо иметь достаточное количество вспомогательных комплексных функций $f_{l, n}^{(\alpha)}$ и параметров $\Omega_{ \pm}$и $Q_{ \pm}$. В частности, стандартный МПР [16] содержит только одну комплексную вспомогательную функцию и два постоянных параметра, которых недостаточно для описания двухкомпонентных уединенных волн $[14,15,17-$ 20], тогда как применение обобщенного уравнения МПР (10) дает нам возможность ввести две комплексные вспомогательные функции и восемь постоянных параметров.

Подводя итог полученным выше результатам, мы делаем следующие выводы: для исследования резонансных, нерезонансных и гибридных ДБ, осциллирующих на СРЧВ, необходимо учитывать производные второго порядка волнового уравнения (9), волны должны быть модулироваными по фазе, т.е. функция $\hat{E}_{l}$ должна быть комплексна (разд. 3), и следует использовать обобщенный ПМР (10).

Известно, что при решении нелинейных дифференциальных уравнений с помощью редукционных методов вводятся вспомогательные функции, с помощью которых исходное уравнение трансформируется к другому уравнению для вспомогательных функций, решение которого хорошо известно. При использовании стандартной версии МПР [16,29] широкий класс нелинейных дифференциальных уравнений сводится для вспомогательной функции к скалярному НУШ, которое имеет солитонное решение. Например, уравнение Синус-Гордон трансформируется к скалярному НУШ и солитонное решение НУШ дает бризерное решение для уравнения Синус-Гордон [30].

Аналогичная ситуация имеет место и для обобщенной версии МПР (10), в рамках которого достаточно широкий класс нелинейных дифференциальных уравнений не только нелинейной оптики, в частности уравнение (9), но и акустики, плазмы, гидродинамики и т.д. трансформируются к связанным НУШ для вспомогательных функции [31,32]. Связанные НУШ и соответствующие решения изучены достаточно подробно. Их называют векторными солитонами или векторными бризерами. Компоненты этих решений могут иметь взаимоперпендикулярные или параллельные поляризации [33,34]. Эти решения зависят от коэффициентов $p_{ \pm}, q_{ \pm}$и $r_{ \pm}$. Так как решаемая нами задача не содержит каких-либо специфических особенностей связанных с НУШ, решения этого уравнения остаются вполне справедливыми и в рассматриваемом случае и не требуется проведение дополнительных исследований. Следует отметить, что иногда рассматривают более сложные версии связанных НУШ, когда эта система уравнений содержит в отличие от уравнений (16) дополнительные члены, связанные, например, с нерезонансными поглощениями, с проводимостью среды, нелокальными эффектами, пространственными неоднородностьями и т. д. Соответствующие решения и свойства этих решений, в том числе 
стабильность, также изучены (см., например, [32,33] и цитируемые там работы).

Гибридный ДБ осциллирующий на СРЧВ уравнение (20), добавляет новые физические условия, позволяющие изучать двухкомпонентную уединенную волну, ранее изучавшаеся только для резонансных и нерезонансных волн. С другой стороны, вместе с гибридными однокомпонентными солитонами и гибридными однокомпонентными бризерами, гибридный ДБ осциллирующий на СРЧВ, делает теорию гибридных нелинейных уединенных волн более полной и релевантной.

\section{Конфликт интересов}

Автор заявляет, что у него нет конфликта интересов.

\section{Список литературы}

[1] Sauter E.G. Nonlinear Optics. Wiley. New York.1996. 186 p.

[2] Виноградова М.Б., Руденко О.В., Сухоруков А.П. // Теория волн. Наука. 1990. 432 с.

[3] Maimistov A.I., Basharov A.M. Nonlinear optical waves. Kluwer Academic Publishers Dordrecht Boston, London. 1999. $643 \mathrm{p}$.

[4] McCall S.L., Hahn E.L. // Phys. Rev.1969. V. 183. P. 457.

[5] Полуэктов И.А., Попов Ю.М., Ройтберг В.С. // УФН. 1974. T. 114. C. 97.

[6] Allen L., Eberly J.H. Optical resonance and two-level atoms. Wiley-Interscience. 1975. 202 р.; Аллен Л., Эберли Дюс. Оптический резонанс и двух-уровневые атомы. М.: Мир. 1978. c. 222.

[7] Schneider P., Borri P., Langbein W., Woggon U., Förstner J., Knorr A., Sellin R.L., Ouyang D., Bimberg D. // Appl. Phys. Lett. 2003. V. 83. P. 3668.

[8] Panzarini G., Hohenester U., Molinari E. // Phys. Rev. B. 2002. V. 65. P. 165322.

[9] Adamashvili G.T., Knorr A. // Optics Letters. 2006. V. 31. P. 74.

[10] Arkhipov M.V., Shimko A.A., Rosanov N.N., Babushkin I., Arkhipov R.M. // Phys. Phys. A. 2020. V. 101. P. 013803.

[11] Ivic Z., Lazarides N., Tsironis G.P. // Chaos, Solitons and Fractals:X. 2019. V. 1. P. 100003.

[12] Sazonov S.V., Ustinov N.V. // Physica Scripta. 2019 V. 94. P. 115208.

[13] Заболотский А.А. // Письма в ЖЭТФ. 2019. Т. 156. С. 890.

[14] Adamashvili G.T. // Results in Physics. 2011. V. 1. P. 26.

[15] Адамашвили Г.Т. // Опт. и спектр. 2012. Т. 113. N 1. С. 83.; Adamashvili G.T. //Opt. Spectrosc. 2012. N 1. P. 81.

[16] Taniuti T., Iajima N. // J. Math. Phys. 1973. V. 14. P. 1389.

[17] Адамашвили Г.Т. // Опт. и спектр. 2018. Т. 125. С. 269.; Adamashvili G.T. // Opt. Spectrosc. 2018. N 2. P. 285.

[18] Адамашвили Г.Т.// Опт. и спектр. 2019. Т. 127. N 11. C. 798.; Adamashvili G.T. //Opt. Spectrosc. 2019. N 5. P. 865.

[19] Adamashvili G.T. // Eur. Phys. J. D. 2020. V. 74. P. 41.

[20] Adamashvili G.T., Adamashvili N.T., Peikrishvili M.D., Koplatadze R.R. // Arxiv:1804.02993v1.

[21] Маймистов А.И., Маныкин Е.А. // ЖЭТФ. 1983. Т. 85. C. 1177.

[22] Nakazawa M., Yamada E., Kubota H. // Phys.Rev. Lett. 1991. V. 66. P. 2625.
[23] Nakazawa M., Yamada E., Kubota H. // Phys. Rev. A. 1991. V. 44. P. 5973.

[24] Caetano D.P., Cavalcanti S.B., Hickmann J.M., Kamchatnov A.M., Kraenkel R.A., Makarova E.A. // Phys. Rev. E. 2003. V. 67. P. 946615.

[25] Adamashvili G.T., Kaup D.J. // Phys. Rev. E. 2004. V. 70. P. 066616.

[26] Fonseca E.J.S., Cavalcanti S.B., Hickmann J.M. // Phys. Rev. E. 2001. V. 64. P. 016619.

[27] Ландау Л.Д., Лифиии, E.M. // Электродинамика сплошных сред. М.: Изд-во техн. теор. лит-ры, 1957. 457 с.

[28] Ландау Л.Д., Лифиии, Е.М. // Квантовая механика. Нерелятивиская теория. М.: Изд-во техн. теор. лит-ры, 1957. $768 \mathrm{c}$.

[29] Leblond H. // J. Phys. B. 2008. V. 41. P. 043001.

[30] Newell A.C. Solitons in Mathematics and Physics. Society for Industrial and Applied Mathematics. 1985. 326 p.

[31] Adamashvili G.T. // arXiv: 2102.04774v1.

[32] Adamashvili G.T. //Chinese Physics B. 2021. V. 30. P. 020503.

[33] Menyuk C.R. // Opt. Lett. 1987. V. 12. P. 614.

[34] Kivshar Y.S., Agrawal G.P. Optical solitons. From Fibers to Photonic Crystals (Academic Press, 2003). 527 p. 